\title{
Neuro-ophthalmic features of carotid cavernous fistulas and their treatment by endoarterial balloon embolisation
}

\author{
D Brosnahan, R M McFadzean, E Teasdale
}

\begin{abstract}
The neuro-ophthalmic features of 11 traumatic carotid cavernous fistulas and their successful occlusion by endoarterial balloon embolisation is reported. Significant improvement in all neuro-ophthalmic signs and symptoms occurred following treatment, however, ocular motility deficits persisted in 7 patients. All 11 fistulas were occluded and the patency of the internal carotid artery was preserved in 9 patients. Though the internal carotid artery was sacrificed in 2 patients there were no permanent sequelae. Transient complications of the procedure occurred in 2 patients.
\end{abstract}

A carotid cavernous fistula (CCF) may occur spontaneously or as a result of trauma. Barrow et al described an anatomical angiographic classification of such fistulas into 4 types. ${ }^{1}$ Type A fistulas are direct high flow shunts between the internal carotid artery (ICA) and the cavernous sinus. Types B-D are dural shunts between meningeal branches of the internal carotid artery, external carotid artery or both and the cavernous sinus. Traumatic fistulas are almost always type A fistulas and rarely resolve without treatment. In type A fistulas trauma produces a rent in the intracavernous portion of the internal carotid artery allowing arterial blood to enter the venous cavernous sinus and its main tributaries, the orbital venous complex, and the inferior petrosal sinus. Raised venous pressure and reduced arterial perfusion cause significant neuro-ophthalmic symptoms and signs. Orbital congestion produces lid swelling, conjunctival and episcleral injection, and proptosis which may be pulsatile. Anterior segment ischaemia may be signified by flare and cells in the anterior chamber, iris atrophy, and secondary glaucoma. Posterior segment changes are characterised by dilated, tortuous retinal veins, and optic disc swelling. Vision may be threatened as a consequence of corneal exposure, secondary glaucoma, or ischaemic optic neuropathy. Ocular motility deficits due to congestion and hypoxia of the extra-ocular muscles or cranial nerve palsies are frequently found. ${ }^{2-4}$

Barrow et al suggest treatment of the CCF is indicated if there is visual deterioration, significant diplopia, intolerable bruit or headache, or proptosis causing untreatable corneal exposure. ${ }^{1}$ The commonly accepted method of treatment is by occlusion of the fistula with a detachable balloon placed in situ via the endo- arterial route. ${ }^{4-6}$ In some patients this approach is not possible and a transvenous approach via the superior ophthalmic vein or inferior petrosal sinus may be successful ${ }^{7}$ Direct surgical approaches to CCFs have been used but have significantly higher rates of failure and morbidity.

\section{Materials and methods}

A retrospective study was performed on patients with traumatic CCFs between 1982-89. A full neuro-ophthalmic assessment was performed before and after treatment where patient cooperation permitted. Angiography was performed to identify the side, site, flow rate, drainage, and any external supply of the fistula. The flow rate was defined as high, moderate or low. A high flow rate occurred where there was no filling of the intracranial vessels beyond the fistula before filling of the fistula. If the anterior and middle cerebral vessels filled at the same time as the fistula, flow was described as moderate (fig 1). A low flow fistula was characterised by normal filling of the anterior and middle cerebral arteries before filling of the fistula.

All patients were treated by transfemoral arterial balloon embolisation which was achieved by introduction of a co-axial catheter system with a detachable latex balloon. ${ }^{5}$ After test occlusion of the fistula the latex balloon was filled with silicone which was allowed to harden before the balloon was detached.

\section{Results}

There were 11 affected patients, 6 females and 5 males. The age range was $12-62$ years with a mean age of 39 years. The time interval between injury and presentation varied between 1 day and 1.5 years.

\section{Aetiology}

Ten patients had a known head injury, 5 resulting from a road traffic accident. One patient, a 12 year old female, had been struck below the eye with a spoke of an umbrella resulting in a skin laceration which bled profusely.

\section{Clinical findings}

Loss of vision occurred in 2 patients being reduced to no perception of light and counting fingers at 1 metre, but it was not possible to record visual acuity in 2 patients due to their decreased conscious level. Proptosis was present in all patients (table 1) and was 


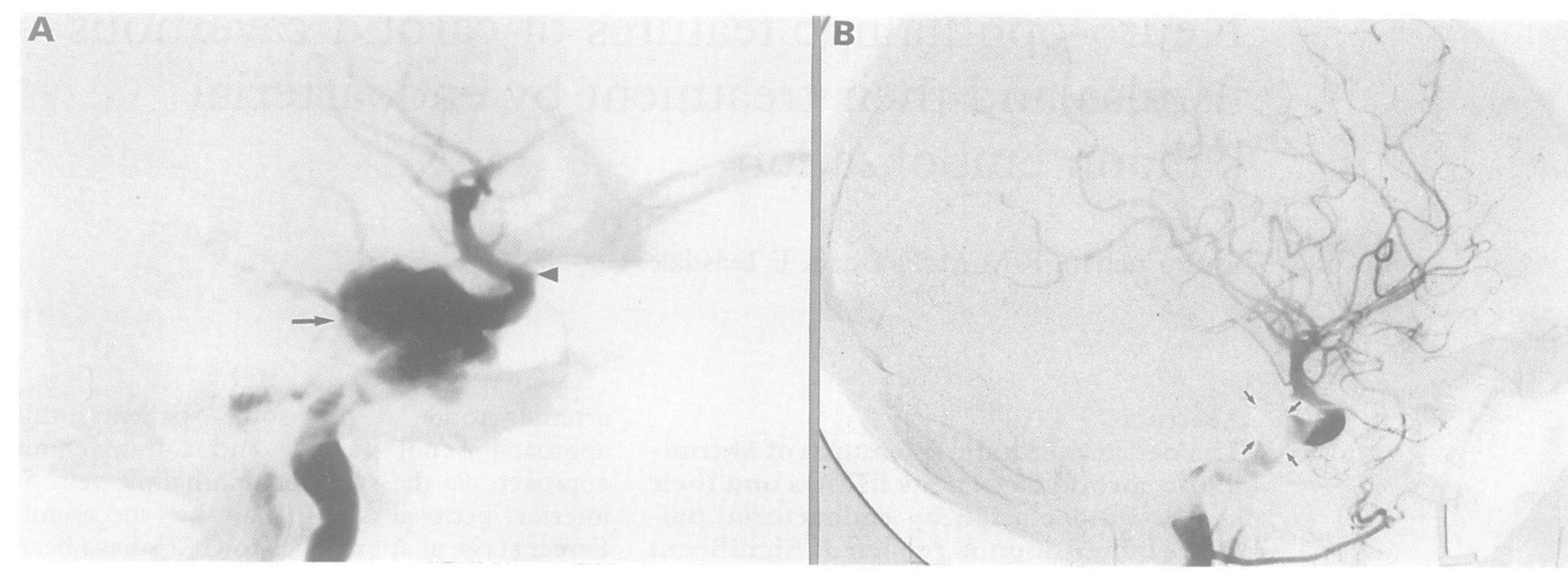

Figure 1 Left, left carotid angiogram demonstrates a carotid cavernous fistula. Contrast is seen to fill the carotid sinus (arrow) and flow in internal carotid artery (arrow head) beyond the fistula as it fills indicates it is a moderate flow fistula; Right, post-embolisation a filling defect (arrows) representing the balloon is seen in the carotid sinus with preservation of the internal carotid artery.

bilateral in 2 patients. Contra-lateral proptosis was found in 1 patient whose angiogram confirmed contra-lateral drainage of the fistula (fig 2).) Dilated tortuous conjunctival vessels were a universal finding as was the presence of a bruit on auscultation. Ten patients had chemosis of the conjunctiva with prolapse of redundant conjunctiva through the interpalpebral aperture in 1 patient. Engorgement of lid vessels more prominent in the upper lid was found in 9 patients. Pulsation of the globe was evident in 5 patients (table 1 ).

The pupils were dilated and unreactive due to a third nerve palsy in 5 patients while a further patient showed a traumatic mydriasis, anterior chamber angle recession, and sequential iris atrophy. Ocular motility deficits were frequent with sixth nerve palsies in 8 patients and third and fourth nerve palsies in 5 patients (Table 2). Reduced sensation in the distribution of the ophthalmic division of the fifth cranial nerve was evident in 3 patients.

The intra-ocular pressure was elevated in 3 patients, ranging from 26 to $30 \mathrm{~mm}$ of mercury. When drainage of the fistula was bilateral the elevated intra-ocular pressure was also bilateral.

Fundoscopy revealed dilated and tortuous retinal veins in 4 patients, with optic disc swelling in 2 patients. There were no significant retinal haemorrhages, exudates or macular changes.

Radiological findings

Angiography showed a CCF on the right side

Table 1 Number of patients with neuro-ophthalmic signs pre and post embolisation

\begin{tabular}{llll}
\hline & Pre-embolisation & $\begin{array}{l}\text { Post-embolisation } \\
\text { Improved }\end{array}$ & $\begin{array}{l}\text { Post-embolisation } \\
\text { Normal }\end{array}$ \\
\hline Decreased Visual Acuity & 2 (1-NPL & - & 1 \\
Proptostis & 11 & 3 & 8 \\
Chemosis & 10 & 1 & 9 \\
Bruit & 11 & - & 11 \\
Pulsation of the globe & 5 & - & 5 \\
Lid engorgement & 9 & - & 9 \\
Venous stasis retinopathy & 4 & - & 4 \\
Disc oedema & 2 & - & 1 \\
Visual loss & 2 & 3 \\
$\uparrow$ IOP & 3 & & \\
\hline
\end{tabular}

in 6 patients and on the left side in 5 patients. The lesion was situated at the junction of the posterior ascending and horizontal segments of the intracavernous carotid artery in 9 patients and in the horizontal segment in 2 patients. Using the criteria described in materials and methods 9 patients had high flow rates and 2 patients had moderate flow rates. Drainage of the fistula was both anterior and posterior in 10 patients, being equal in both directions in 6 patients, but predominantly anterior in 4 patients. One fistula drained only anteriorly. Bilateral drainage occurred in 2 patients, 1 of whom showed normal venous drainage on the side of the fistula with a markedly dilated and tortuous orbital venous system on the contralateral side (fig 2). No feeder vessels from the external carotid artery were identified in any of the cases.

\section{Interventional radiology}

The CCFs were successfully closed by transarterial balloon embolisation in all patients. One balloon was sufficient to occlude the fistula in 8 patients while 2 patients each required 2 and 3 balloons, respectively. In 1 patient the CCF closed spontaneously during balloon manipulation. The patency of the internal carotid artery was preserved in 9 patients $(82 \%)$ but in 2 patients $(18 \%)$ it was necessary to occlude the internal carotid artery to effect closure of the fistula.

Transient complications of the procedure occurred in 2 patients. In 1 patient an initial failed attempt at closure resulted in worsening of the orbital signs. However, the fistula was closed successfully 2 days later although the internal carotid artery was sacrificed. The second patient developed a grand mal fit with aphasia and contralateral hemiparesis 12 hours after embolisation, but complete resolution took place within 1 week.

\section{Clinical sequelae}

After closure of the fistula, signs resulting from engorgement of the orbital contents showed most improvement. Proptosis was absent in $\mathbf{8}$ patients but persisted in a mild degree in 3 

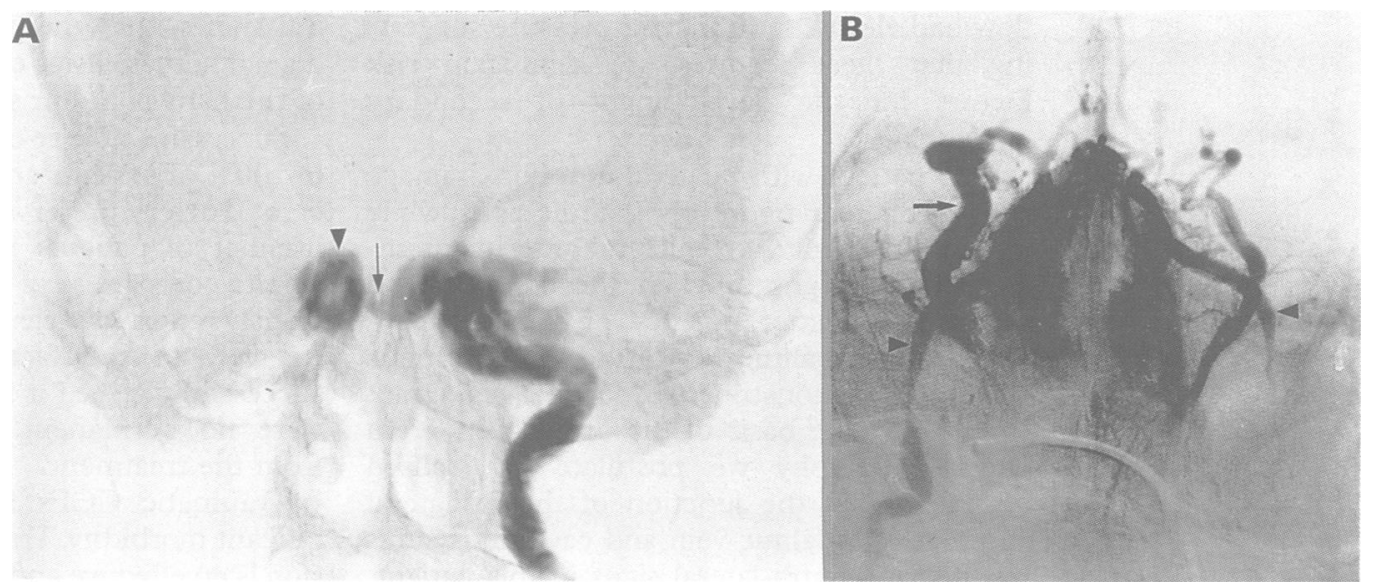

Figure 2 Left, Left carotid angiogram demonstrates the left carotid cavernous fistula, the anterior communicating vein (arrow) and the right cavernous sinus (arrow head); Right, orbital venogram in the same patient demonstrates the patency of both superior ophthalmic veins, however, the right (arrow) is seen to be dilated. Both facial veins are demonstrated (arrow head).

patients. Despite angiographic evidence of fistula occlusion, a mild degree of conjunctival injection and swelling persisted in 1 patient. In all cases the bruit and pulsations of the globe resolved. Visual acuity returned to $6 / 6$ (Snellen type) in 1 patient with pre-treatment acuity of counting fingers at 1 metre, but the patient with no perception of light showed no improvement and optic atrophy supervened. Intra-ocular pressure returned to within normal limits in the 3 affected patients while retinal vascular changes and disc swelling showed complete resolution (table 1).

Cranial nerve palsies and extra-ocular muscle dysfunction proved most resistant to resolution. Of 8 patients with a sixth nerve palsy, 4 patients showed complete recovery with partial recovery in 1 patient and no improvement in 3 patients. Third nerve palsies completely recovered in 1 patient, improved in 2 patients and were unchanged in 2 patients. Fourth nerve palsies resolved completely in 3 patients, improved in 1 patient, and persisted in 1 patient (Table 2). Paraesthesia in the distribution of the ophthalmic division of the fifth cranial nerve resolved in 2 patients but persisted in 1 patient.

\section{Discussion}

Traumatic CCFs are generally high flow fistulas due to a severe head injury. Occasionally apparently trivial trauma, as in the 12 year old girl whose cavernous sinus was penetrated by the spoke of an umbrella passing along the orbital floor, may result in fistula formation. A similar case of traumatic CCF is described in a five year old girl who had been struck in the eye by a fishing rod. ${ }^{5}$ In this series there was no sex predilection although the previous studies have shown a strong male preponderance. ${ }^{9}$ The

Table 2 Number of patients with cranial nerve palsies pre and post embolisation

\begin{tabular}{llll}
\hline & Pre-embolisation & $\begin{array}{l}\text { Post-embolisation } \\
\text { Improved }\end{array}$ & $\begin{array}{l}\text { Post-embolisation } \\
\text { Normal }\end{array}$ \\
\hline III Nerve & 5 & 2 & 1 \\
IV Nerve & 5 & 1 & 3 \\
VI Nerve & 8 & 1 & 4 \\
\hline
\end{tabular}

mean age of 39 years reflects the propensity of younger persons to sustain severe head injuries, particularly in road traffic accidents. ${ }^{10}$

The incidence of visual loss complicating CCF has been reported to vary between $21 \%$ and $63 \%$ of cases, ${ }^{2311}$ commonly due to traumatic and/or ischaemic optic neuropathy. Visual loss may also result from corneal exposure, secondary glaucoma, hypoxic retinopathy or rupture of the globe. Loss of vision was permanent in 1 patient who presented with no perception of light in the affected eye and subsequently developed optic atrophy. In a second patient with marked proptosis, disc swelling and venous stasis retinopathy vision was reduced to counting fingers at 1 metre, but recovered to $6 / 6$ (Snellen type) following closure of the fistula.

The reported incidence of secondary glaucoma varies between $21 \%$ and $70 \%$ and is often refractory to medical treatment. ${ }^{1-4}$ In this series 3 patients had intra-ocular pressure within the range of 28 to $30 \mathrm{~mm} \mathrm{Hg}$, which returned to normal levels following occlusion of the fistula.

Extra-ocular muscle dysfunction was common, occurring in 9 of 11 patients, and may result from a cranial nerve palsy (traumatic and/or ischaemic) or hypoxic congestion of the extra-ocular muscles. Of the 8 patients with a sixth nerve palsy, 2 patients developed their palsy and CCF some months after the initial injury, suggesting insult to the nerve in the cavernous sinus or Dorello's canal. In both cases there was incomplete resolution following closure of the fistula. Detection of a third nerve palsy in the congested orbit is difficult because the ptosis which ensues may be mechanical or neurogenic and iris ischaemia renders pupillary responses unreliable. In this series all third nerve palsies dated from the time of initial injury, thus making comment on their aetiology problematic. Defective ocular motility was the most frequent and disabling sequel, persisting in 7 patients (table 2).

Greater anterior drainage of the CCF occurred in 4 patients of whom 3 had high flow fistulas, the remaining being moderate flow. Those patients with anterior drainage and high 
flow had elevated intraocular pressure suggesting that these features are significant risk factors for the development of secondary glaucoma.

One patient with bilateral anterior drainage showed bilateral neuro-ophthalmic signs, while a second patient with bilateral anterior drainage showed signs only on the contra-lateral side. In the latter patency of the ipsilateral superior ophthalmic vein up to the cavernous sinus was demonstrated by orbital venogram (fig 2). On the basis of the orbital venogram and angiography we postulate a localised obstruction at the junction of the ipsilateral superior ophthalmic vein and cavernous sinus resulted in contra-lateral signs in this patient. An obvious prerequisite for a contra-lateral drainage is patency of the inter-cavernous sinuses. Thirteen cases with contra-lateral signs have been described and it has been proposed that contra-lateral drainage follows thrombosis of the ipsilateral superior ophthalmic vein and inferior petrosal sinus. ${ }^{12} \mathrm{Nec}-$ ropsy evidence showed thrombosis of the ipsilateral superior ophthalmic vein in $1 \mathrm{case}^{13}$ and an aneurysm of the carotid syphon compressing communication between the cavernous sinus and the superior ophthalmic vein in another case. ${ }^{14}$

The latex balloons were inflated with silicone as deflation is more frequent with the use of contrast and may result in balloon dislodgement or surrounding false aneurysm formation. ${ }^{1516}$ Closure of the fistula during attempted embolisation without placement of a balloon was observed in one patient. This phenomenon has previously been described, ${ }^{17}$ probably due to a change in the flow pattern and temporary occlusion during the procedure, and leads to complete thrombosis.

Transient complications following treatment occurred in 2 patients due to presumed middle cerebral artery embolism from a carotid atheromatous plaque in one patient and an initial failed attempt at closure followed by successful secondary intervention with internal carotid artery occlusion in a second patient. The other patient in whom the carotid artery was occluded had a large intracranial pseudo-aneurysm associated with the CCF and it was not possible to occlude the fistula without also occluding the carotid artery. Neither patient showed signs of cerebral ischaemia following occlusion of the carotid artery.

Permanent and transient cranial nerve palsies complicating treatment are reported in some series. In a review of the literature Keltner ${ }^{18}$ noted permanent cranial nerve palsies in $3-30 \%$ of patients and transient nerve palsies in $3-67 \%$ of patients as a result of embolisation. The third cranial nerve was most commonly involved, the proposed mechanism being compression by the balloon during inflation within the cavernous sinus. ${ }^{1819}$ No cranial nerve palsies complicated embolisation of the fistulas in our series.

Successful closure of the fistula was achieved in $100 \%$ of patients and patency of the internal carotid artery preserved in $82 \%$. Although the number of patients is relatively small, these results compare very favourably with the previously reported series in both respects. ${ }^{4-6}$ 17-19 In view of the fact that all fistulas were successfully closed it is encouraging that there were no permanent complications resulting from the treatment.

Traumatic CCFs frequently result in significant morbidity. Transarterial balloon occlusion is an effective and relatively safe method of treating such fistulas. Permanent visual loss occurred in only 1 patient, but significant ocular motility deficits secondary to cranial nerve palsies proved to be the most frequent and persistent complication of CCFs.

1 Barrow DL, Spector RH, Braun IF, et al. Classification and treatment of spontaneous carotid cavernous fistulas. Neurosurg 1985;62:248-56.

2 Sanders MD, Hoyt WF. Hypoxic sequel of carotid cavernous fistulae. Br $\mathcal{Y}$ Ophthalmol 1969;53:82-97.

3 Leonard TJK, Moseley IF, Sanders MD. Ophthalmoplegia in carotid cavernous sinus fistula. $B r f \mathcal{f}$ Ophthalmol in carotid cavernot

4 Kupersmith MJ, Bernstein A, Flamm E, et al. Neuroophthalmic abnormalities and intravascular therapy of traumatic carotid cavernous fistulas. Ophthalmology

5 Debrun GM, Lacour P, Vinvela F, et al. Treatment of 54 traumatic carotid cavernous fistulas. $f$ Neurosurg 1991;55:678-92.

6 Debrun GM, Vinvela F, Fox AJ, et al. Indications of treatment from classification of 132 carotid-cavernous fistulae. Neurosurgery 1988;22:285-9.

7 Hanneken AM, Miller NR, Debrun GM, et al. Treatment of carotid cavernous sinus fistulas using a detachable balloon catheter through the superior ophthalmic vein. Arch Ophthalmol 1989;107:87-92.

8 Dolenc V. Direct micro-surgical repair of intracavernous vascular lesions. I Neurosurg 1983;58:824-31.

9 Jennett B, Teasdale G, Galbraith S, et al. Severe head injuries in three countries. I Neurol Neurosurg Psychiatry injuries in three

10 Jennett B, Teasdale G, Braakman R, et al. Prognosis of patients with severe head injury. Neurosurgery 1979; 4:283-9.

11 Palestine AG, Younge BR, Piepgras DG. Visual prognosis in carotid cavernous fistula. Arch Ophthalmol 1981 99:1600-3.

12 Theron J, Olivier A, Melancon A, et al. Left carotidocavernous fistula with right exophthalmos: treatment by detachable balloon. Neuroradiology 1985;27:349-53.

13 Dandy WE, Follis RH. On pathology of carotidocavernous aneurysms (pulsating exophthalmos). Am f Ophthalmol 1941;24:365-85.

14 Tamler E. Carotid cavernous fistula with typical signs on contralateral side only. Arch Ophthalmol 1954;52:433-41.

15 Quisling RG, Mickle JP, Ballinger JR, et al. Latex vascular occlusion balloons. Histopathologic evaluation in a high flow autocaval fistula model. Am $f$ Neuroradiol 1985; 6(4):583-7.

16 Scialfa G, Valsecchi F, Scotti G. Treatment of vascular lesions with balloon catheters. Am $\mathcal{F}$ Neuroradiol 1983 4(3):395-8

17 Berthelsen B, Svendsen P. Treatment of carotid cavernous fistulas with detachable balloons. Acta Radiol 1987 28(6):683-91.

18 Keltmer JL, Sattersfield D, Dublin AB, et al. Dural and carotid cavernous sinus fistulas. Ophthalmol 1987 94:1585-600.

19 Debrun WE. Treatment of carotid cavernous sinus and vertebral fistulae by detachable balloons. Review of 43 cases. Presented at the Annual Meeting of the American Association of Neurological Surgeons, New York, April 1980. 